\title{
Degradation of paracetamol by pure bacterial cultures and their microbial consortium
}

\author{
Lili Zhang • Jun Hu • Runye Zhu • Qingwei Zhou • \\ Jianmeng Chen
}

Received: 7 March 2012 / Revised: 25 April 2012 /Accepted: 10 May 2012 /Published online: 31 May 2012

(C) Springer-Verlag 2012

\begin{abstract}
Three bacterial strains utilizing paracetamol as the sole carbon, nitrogen, and energy source were isolated from a paracetamol-degrading aerobic aggregate, and assigned to species of the genera Stenotrophomonas and Pseudomonas. The Stenotrophomonas species have not included any known paracetamol degraders until now. In batch cultures, the organisms $\mathrm{fl}, \mathrm{f} 2$, and fg- 2 could perform complete degradation of paracetamol at concentrations of $400,2,500$, and 2,000 mg/L or below, respectively. A combination of three microbial strains resulted in significantly improved degradation and mineralization of paracetamol. The co-culture was able to use paracetamol up to concentrations of $4,000 \mathrm{mg} / \mathrm{L}$, and mineralized $87.1 \%$ of the added paracetamol at the initial of $2,000 \mathrm{mg} / \mathrm{L}$. Two key metabolites of the biodegradation pathway of paracetamol, 4aminophenol, and hydroquinone were detected. Paracetamol was degraded predominantly via 4-aminophenol to hydroquinone with subsequent ring fission, suggesting new pathways for paracetamol-degrading bacteria. The degradation of paracetamol could thus be performed by the single isolates, but is stimulated by a synergistic interaction of the
\end{abstract}

Electronic supplementary material The online version of this article (doi:10.1007/s00253-012-4170-5) contains supplementary material, which is available to authorized users.

L. Zhang $\cdot$ J. Hu $\cdot$ R. Zhu $\cdot$ Q. Zhou $\cdot$ J. Chen $(\bowtie)$

School of Biological and Environmental Engineering,

Zhejiang University of Technology,

No.6 District, Zhaohui,

Hangzhou 310032, China

e-mail: jchen@zjut.edu.cn

\section{Zhang}

State Key Laboratory of Environmental Aquatic Chemistry, Research Center for Eco-Environmental Sciences, Chinese Academy of Sciences,

Beijing 100085, China three-member consortium, suggesting a possible complementary interaction among the various isolates. The exact roles of each of the strains in the consortium need to be further elucidated.

Keywords Biodegradation $\cdot$ Paracetamol $\cdot$ Metabolite . Pathway

\section{Introduction}

Pharmaceuticals are subclasses of organic contaminants that have been detected in wastewater and surface waters throughout the world (Benotti et al. 2009; Philips et al. 2010; Rosal et al. 2008). These emerging pollutants are continuously introduced into the aquatic environment by several emissions from manufacturing facilities, consumer use and disposal, and hospital waste (Langford and Thomas 2009; Philips et al. 2010). Even though the detected concentrations are typically in the nanogram- to microgram-perliter range, it cannot be excluded that molecules designed to be biologically active affect sensitive aquatic organisms even at such low concentrations (Huber et al. 2005). A variety of potential adverse effects including acute and chronic damage, accumulation in tissues, reproductive damage, inhibition of cell proliferation, and behavioral changes have been documented in these low levels (Escher et al. 2011; Philips et al. 2010). This makes essential the development of innovative and cost-effective approaches to ensure the total destruction of pharmaceuticals for the further protection of water quality and ecological health.

Paracetamol (4-acetaminophenol) is a widely used analgesic and antipyretic drug, and an important material for the manufacturing of azo dyes and photographic chemicals. It has been found with a concentration of up to $6 \mu \mathrm{g} / \mathrm{L}$ in 
European sewage effluents (Ternes 1998), up to $10 \mu \mathrm{g} / \mathrm{L}$ in USA natural waters (Kolpin et al. 2002), and more than $65 \mu \mathrm{g} /$ $\mathrm{L}$ in Tyne river in the UK (Robert and Thomas 2006). It may enter the environment mainly from manufacturing wastes (Philips et al. 2010; Skoumal et al. 2006). Currently, paracetamol wastewater is mainly treated by chemical oxidation processes such as electrochemical (Brillas et al. 2005), ozonation (Andreozzi et al. 2003), $\mathrm{H}_{2} \mathrm{O}_{2} / \mathrm{UV}$ oxidation (Andreozzi et al. 2003; Skoumal et al. 2006), $\mathrm{TiO}_{2}$ photocatalysis (Yang et al. 2008, 2009), and solar photoelectro-Fenton oxidation (Li et al. 2007). Although these chemical methods may be available for treating these pollutants, the harsh reaction conditions, the generation of secondary pollutants, and the high operational cost associated with these methods have often made them not a desirable choice (Chen et al. 2010; Li et al. 2007). Biodegradation of pharmaceutical compounds is being considered as an environmentally friendly and low-cost option, and has been demonstrated to have the potential to eliminate pharmaceuticals by degrading them into innocuous end products such as $\mathrm{CO}_{2}$ and $\mathrm{H}_{2} \mathrm{O}$ (Hasan et al. 2011; Chen et al. 2010). However, little information is available about the biodegradation of paracetamol compared to other pharmaceutical compounds. To our knowledge, the only published reports of paracetamol biodegradation were that of Shariati et al. (2010) and that of Gusseme et al. (2011), who reported the removal of paracetamol by a membrane bioreactor. To date, only two pure cultures, Delftia tsuruhatensis and Pseudomonas aeruginosa, were isolated and identified as paracetamol-degrading strains (Gusseme et al. 2011).

In the previous work, we have demonstrated the removal of paracetamol by a microbial aggregate (Hu et al. 2012). In the present study, pure cultures and bacterial consortium able to degrade paracetamol as the sole source of carbon, nitrogen, and energy were isolated from the previous microbial aggregate. The biodegradation efficiency, degradation and mineralization extent, kinetics, and metabolic pathway of paracetamol were determined to provide useful clues for further application in the engineered bioremediation systems. Furthermore, the degradative capacity of the consortium was compared to that of each of the strains individually with regard to the ability to degrade and mineralize paracetamol under the same conditions. These studies are prerequisities to the development of commercial systems to treat paracetamol-contaminated wastewaters.

\section{Materials and methods}

Chemicals and cultivation medium

Paracetamol, hydroquinone, 4-aminophenol, and catechol were obtained from Sigma-Aldrich China, Inc. (Shanghai, China). All other chemicals were of the highest purity commercially available.
The applied mineral salts medium (MSM) contained the following composition: per liter of deionized water, $0.5 \mathrm{~g}$ of $\mathrm{KH}_{2} \mathrm{PO}_{4}, 0.5 \mathrm{~g}$ of $\mathrm{K}_{2} \mathrm{HPO}_{4}, 0.01 \mathrm{~g}$ of $\mathrm{NaCl}, 0.2 \mathrm{~g}$ of $\mathrm{MgCl}_{2} \cdot 6 \mathrm{H}_{2} \mathrm{O}, 0.02 \mathrm{~g}$ of $\mathrm{CaCl}_{2}, 0.339 \mathrm{mg}$ of $\mathrm{MnSO}_{4}$, $0.428 \mathrm{mg}$ of $\mathrm{ZnSO}_{4}, 0.347 \mathrm{mg}$ of $\left(\mathrm{NH}_{4}\right)_{6} \mathrm{Mo}_{7} \mathrm{O}_{24} \cdot 4 \mathrm{H}_{2} \mathrm{O}$, $0.4 \mathrm{mg}$ of $\mathrm{CoCl}_{2} \cdot 6 \mathrm{H}_{2} \mathrm{O}$, and $10 \mathrm{mg}$ of EDTA. After adjusting the $\mathrm{pH}$ to 7.2 to 7.4 with $8 \mathrm{M} \mathrm{NaOH}$, the solution was autoclaved at $121^{\circ} \mathrm{C}$ for $15 \mathrm{~min}$. Paracetamol was added as the sole carbon and nitrogen sources.

\section{Origin and isolation of strains}

Strains were isolated from a previous paracetamol-degrading microbial aggregate in a lab-scale airlift sequencing batch reactor (Hu et al. 2012). The paracetamol-degrading bacteria harvested from the reactor were further cultivated in a batch culture fed with MSM containing paracetamol as the sole sources of carbon, nitrogen, and energy. Strains were isolated on solid media consisting of mineral salts medium, $1.5 \%$ agar, and $500 \mathrm{mg} / \mathrm{L}$ paracetamol, which were added as a sterile solution to the autoclaved media. The plates showed different colony morphologies. Eight different colony types were picked up and plated on Luria-Bertani medium. Finally, these isolates were subcultured in test tubes with liquid MSM medium containing $500 \mathrm{mg} / \mathrm{L}$ paracetamol. Three isolates were able to degrade paracetamol.

Strains were identified according to its phenotypic and genotypic characteristics. Preliminary characterization was based on morphological, physiological, and biochemical tests. Further identification was completed by 16S RNA gene sequencing and GN and GP microplates (Biolog, Inc., Hayward, CA).

\section{Chemical analysis}

Paracetamol utilization was analyzed using a reverse-phase HPLC (Agilent Technologies 1200 Series) equipped with a UV detector (at $240 \mathrm{~nm}$ ). The separation was performed at $30{ }^{\circ} \mathrm{C}$ on an Eclipse XDB-C18 column $(5 \mu \mathrm{m}, 4.6 \times$ $150 \mathrm{~mm}$ ). The mobile phase consisted of $\mathrm{CH}_{3} \mathrm{OH} / \mathrm{H}_{2} \mathrm{O}$ (ratio $15 / 85$ ) with a flow rate of $0.8 \mathrm{~mL} / \mathrm{min}$. The retention time of paracetamol under these conditions was $6.492 \mathrm{~min}$. 4Aminophenol and hydroquinone as possible metabolic intermediates of paracetamol were also detected by the same HPLC method as described above.

The total organic carbon (TOC) concentration was measured on the filtrates using a TOC/TN-VCSH analyzer (Shimadzu, Japan) to determine the mineralization degree of the organic pollutants during the biodegradation process. Microbial growth was monitored by measuring the optical density in a $1-\mathrm{cm}$ cell at $600 \mathrm{~nm}$ on a Hitachi U-2910 spectrophotometer (Hitachi High Technologies America, Inc.). The absorbance was correlated with dry weight to determine the 
actual biomass concentration. Biomass dry weight was determined after cell suspensions were filtered and dried $(24 \mathrm{~h}$, $105^{\circ} \mathrm{C}$ ) with glass fiber paper $>1 \mu \mathrm{m}$ retentivity (Schleicher \& Schuell, Dassel, Germany).

\section{Identification of metabolites}

Biodegradation products were analyzed and tentatively identified by means of gas chromatography-mass spectrometry (GC-MS), liquid chromatography-mass spectrometry (LC-MS), and ion chromatography (IC), and the paracetamol degradative pathway was also proposed based on the identification of metabolic intermediates.

Cells of strains were grown overnight in MSM medium with paracetamol as the sole carbon and energy sources. A resting cell reaction mixture was made by resuspending washed cells from the overnight culture in $25 \mathrm{ml}$ of $40 \mathrm{mM}$ sodium phosphate buffer ( $\mathrm{pH}$ 7.2), and the suspension was stirred at $28{ }^{\circ} \mathrm{C}$. The reaction was initiated by addition of paracetamol to a final concentration of $500 \mathrm{mg} /$ L. After $2 \mathrm{~h}$, the reaction was stopped by rapidly chilling the suspension in an ice water bath. Cells were removed by centrifugation, and the supernatant was extracted three times with equal volumes of ethyl acetate. Combined extracts were dried over anhydrous sodium sulfate and evaporated to dryness by a stream of nitrogen.

Trimethylsilyl (TMS) derivatives were formed by using Regisil RC-2 [N,O-bis (trimethylsilyl)-trifluroacetamide] with $1 \%$ trimethylchlorosilane. Samples were dissolved in $25 \mu \mathrm{l}$ of pyridine, mixed with $50 \mu \mathrm{l}$ of Regisil RC-2, and heated at $60{ }^{\circ} \mathrm{C}$ for $2 \mathrm{~h}$.

GC-MS (Agilent 7890N/MS 5975) equipped with a DB-5 capillary column $(30 \mathrm{~m} \times 0.25 \mathrm{~mm} \times 0.25 \mu \mathrm{m}$, J\&W Scientific, USA) was used for analyzing all derivative metabolites. Conditions for GC-MS were as follows: oven temperature $7{ }^{\circ} \mathrm{C}$ for $5 \mathrm{~min}$, ramped to $280{ }^{\circ} \mathrm{C}$ at $5{ }^{\circ} \mathrm{C} / \mathrm{min}$, and held for 5 min. Injector temperature was $180{ }^{\circ} \mathrm{C}$. The ionization source temperature was set at $220{ }^{\circ} \mathrm{C}$. The mass spectra were obtained at $70 \mathrm{eV}$. All metabolites were identified by GC-MS by matching retention times and ion spectra with authentic standard and NIST library data.

HPLC-MS (Agilent 1200/Therm LCQ TM Deca XP plus) was also tentatively employed for identification of degradation products. The HPLC-MS analysis was carried out on an ion trap mass spectrometer mentioned above. The ESI source voltage was kept at $3.5 \mathrm{kV}$ and capillary temperature at $275{ }^{\circ} \mathrm{C}$. Nitrogen was used as both sheath gas.

Nitrite, nitrate, formic acid, and succinic acid were determined by an ion chromatography (ICS-2000, Dionex) after centrifugations of the samples at $5,000 \times \mathrm{g}$ for $10 \mathrm{~min}$ and filtering through a $0.22-\mu \mathrm{m}$ filter. An IonPac AS19 (250× $4 \mathrm{~mm}$; Dionex) column was used for anion separation.

\section{Oxygen consumption}

The rate of oxygen consumption by strains $\mathrm{f} 1, \mathrm{f} 2$, and fg2 with different initial concentrations of paracetamol was monitored using a Clark-type oxygen electrode system (Oxytherm, Hansatech, UK). The electrode was kept at $28{ }^{\circ} \mathrm{C}$. The well of the oxygen electrode was filled with $2.5 \mathrm{~mL}$ of aerated $50 \mathrm{mM}$ phosphate $(\mathrm{pH} 7.0)$ buffer and $100 \mu \mathrm{L}$ of cell suspension, and the rate of oxygen consumption without substrate (the endogenous consumption) was then measured over at least $5 \mathrm{~min}$. The oxygen consumption rate of substrate was then measured over at least $5 \mathrm{~min}$. The difference between this rate and the endogenous rate was taken to be the increase in oxygen consumption due to the substrate. Experiments were performed in duplicate.

\section{Kinetics experiments}

Batch experiments were conducted to investigate the effect of initial concentrations of paracetamol on the growth kinetics of the three isolates. Each batch degradation was carried out in sterilized $500-\mathrm{mL}$ Erlenmeyer flasks as described above. Bottles were amended with various concentrations of paracetamol and inoculated with the same biomass amounts. Bottles were incubated at $30{ }^{\circ} \mathrm{C}$ on a rotary shaker at $200 \mathrm{rpm}$. Several kinetics models were fitted to the experimental data for selecting the best models. Out of the models, Haldane's growth model was selected due to its mathematical simplicity and wide acceptance for representing the growth kinetics of inhibitory substrates. The Haldane's inhibitory growth kinetics equation is as follows:

$\mu=\frac{1}{X} \frac{d X}{d t}=\frac{\mu_{\max } S}{K_{\mathrm{s}}+S+S^{2} / K_{i}}$

where $S$ is the substrate concentration, $\mu$ is specific growth rate (in per hour), $X$ is the cell mass concentration, $K_{\mathrm{s}}$ is the half saturation coefficient (in milligrams per liter), and $K_{i}$ is an inhibition constant (in milligrams per liter).

Nucleotide sequence accession number

The complete sequences of the 16S rRNA of strains f1, $\mathrm{f} 2$, and fg-2 have been deposited in the GenBank database under accession nos. JQ717287, JQ717288, and JF357964, respectively. The strains have been deposited in China Center for Type Culture Collection (Wuhan) under accession number CCTCC AB 2012008, CCTCC AB 2012007, and CCTCC AB 2012009 for f1, f2, and fg-2, respectively. 


\section{Results}

Isolation and identification

The bacterial strains f1, f2, and fg-2 able to degrade paracetamol as the sole source of carbon, nitrogen, and energy were isolated from a previous microbial aggregate cultivated in a sequencing batch reactor for treating paracetamolcontaminated wastewater ( $\mathrm{Hu}$ et al. 2012). Strain f1 was identified as a Stenotrophomonas sp., and strains $\mathrm{f} 2$ and fg-2 as Pseudomonas sp. The $16 \mathrm{~S}$ rRNA gene sequence of strain f1 (JQ717287) shows $99 \%$ identity to the $16 \mathrm{~S}$ rRNA gene sequence of Stenotrophomonas maltophilia strain C-JPDA6 (HM755607). Strain f2 (JQ717288) had $99 \%$ sequence identity to those of Pseudomonas sp. SMIC-3 (FJ877154). Phylogenetic analysis for the sequence of $\mathrm{fg}-2$ 16S rRNA (JF357964) shows that fg-2 also belonged to the genus Pseudomonas. Meanwhile, the isolates were also identified by carbon substrate oxidation patterns using Biolog-GN test. According to the metabolic fingerprinting (data not shown), f1 was also assigned to the genus Stenotrophomonas after 5-h incubation with probability, similarity (SIM) index, and genetic distance (DIS) of $98.6 \%$, 0.796 , and 3.06 , respectively. F2 had $99.2 \%$ identity to Pseudomonas sp. with SIM and DIS of 0.80 and 4.1, respectively. The metabolic fingerprinting of fg-2 shows $98.3 \%$ identity to Pseudomonas sp. with appropriate DIS and SIM values. Consequently, both $16 \mathrm{~S}$ rRNA sequence and Biolog-GN tests clearly showed that strains f1 belonged to Stenotrophomonas sp., while both $\mathrm{f} 2$ and fg- 2 belonged to Pseudomonas species.

\section{Biodegradation and mineralization of paracetamol}

Paracetamol degradation and mineralization by each isolated strain and their mixed bacterial consortium were evaluated at initial paracetamol concentrations from 100 to $4,500 \mathrm{mg} / \mathrm{L}$. Figure 1 shows the paracetamol concentration profiles with respect to incubation time and paracetamol mineralization on the basis of TOC removals.

Three isolated strains, f1, f2, and fg-2, could grow on paracetamol as the sole carbon, nitrogen, and energy source (Fig. 1a). For f1, a complete reduction was achieved at about $116 \mathrm{~h}$ at initial concentration of $400 \mathrm{mg} / \mathrm{L}$. When the concentration of paracetamol was increased to $600 \mathrm{mg} / \mathrm{L}$, only $70 \%$ of paracetamol added was degraded within $150 \mathrm{~h}$, suggesting paracetamol concentrations in excess of $600 \mathrm{mg} / \mathrm{L}$ would inhibit the growth of fl strongly. Strain f2 was capable of performing complete degradation of paracetamol at about $60 \mathrm{~h}$ at initial concentration of $2,000 \mathrm{mg} / \mathrm{L}$ or at about $70 \mathrm{~h}$ at initial concentration of $2,500 \mathrm{mg} / \mathrm{L}$. At $3,000 \mathrm{mg} / \mathrm{L}$, paracetamol degradation was completely inhibited. Strain fg-2 could degrade paracetamol up to
2,000 mg/L completely within $45 \mathrm{~h}$. Based on paracetamol concentrations at the exponential phase, the paracetamol removal rate constants for the batch experiments were calculated by performing a linear regression analysis [see Fig S-1, Electronic supplementary material (ESM)]. The results suggested that the degradation rate of paracetamol by $\mathrm{f1}, \mathrm{f} 2$, and $\mathrm{fg}-2$ was in the order of $\mathrm{fg}-2>\mathrm{f} 2>\mathrm{f} 1$.

The degradative capacity of the consortium including $\mathrm{fl}$, f2, and fg-2 was compared to that of each of the strains individually with regard to the ability to degrade paracetamol at the same concentration ranges. It should be noted that combining strains $\mathrm{f} 1, \mathrm{f} 2$, and fg-2 significantly improved the paracetamol biodegradation. The consortium was able to utilize paracetamol up to concentrations of $4,000 \mathrm{mg} / \mathrm{L}$ completely, and resulted in rapid degradation of paracetamol. For example, at an initial paracetamol concentration of $2,000 \mathrm{mg} / \mathrm{L}$, the degradation reached $100 \%$ within $37 \mathrm{~h}$, and the lag time was reduced to $8 \mathrm{~h}$, suggesting a substantially higher degradation efficiency and significantly better tolerance to paracetamol than that of the individual strains. Namely, the co-culture of $\mathrm{f1}, \mathrm{f} 2$, and fg-2 performed better than each strain on the paracetamol degradation. These data demonstrated a possible synergistic interaction among f1, $\mathrm{f} 2$, and fg-2 took place in the paracetamol degradation.

The change of TOC is a good indicator of mineralization for the overall organic contents including parent and daughter compounds. The mineralization extent was calculated and compared with the biodegradation of paracetamol by individual strains and their consortium as shown in Fig. 1b. As expected, the co-culture mineralized significantly more paracetamol than did pure strains in all cases and to various extents. At a paracetamol concentration of $400 \mathrm{mg} / \mathrm{L}$, TOC removals of $\mathrm{fl}, \mathrm{f} 2, \mathrm{fg}-2$ and their consortium were $65,84.5$, 85.9 , and $97.9 \%$, respectively. The $\mathrm{f} 2$ and fg- 2 mineralized about 65.2 and $48.9 \%$ of the added paracetamol at initial concentration of $2,000 \mathrm{mg} / \mathrm{L}$. For the co-culture of $\mathrm{f1}, \mathrm{f} 2$, and fg-2, the removal of TOC was up to $87.1 \%$. These data suggest that a combination of three microbial strains recovered from the same culture was shown to be necessary for paracetamol mineralization to a great extent.

\section{Growth kinetics}

The growth kinetics of strains $\mathrm{fl}, \mathrm{f} 2$, and fg- 2 and their consortium were determined using Haldane's growth kinetics model, which is a modified version of the Monod kinetics taking into account an inhibition factor. The relationship between specific growth rate and paracetamol concentration is shown in Fig. 2. The specific growth rate increased with increases in paracetamol concentration and reached a maximum at approximately $100,725,750$, and $1,000 \mathrm{mg} / \mathrm{L}$ for $\mathrm{f1}, \mathrm{f} 2$, fg-2, and their consortium, respectively. Concentrations above these levels decreased the specific growth rates 
a

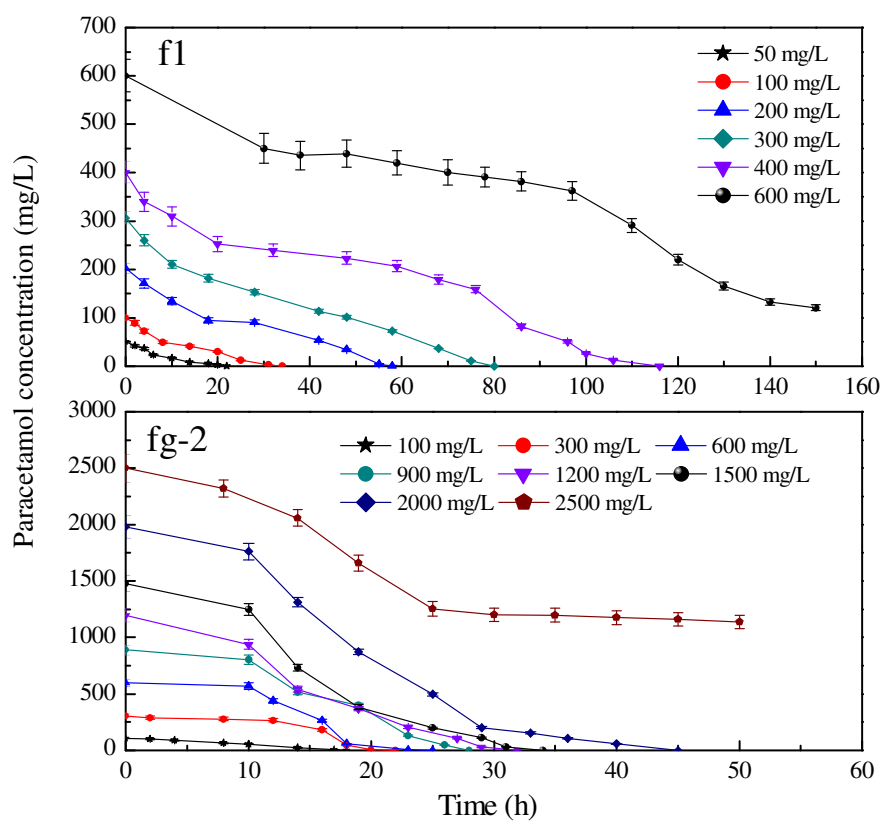

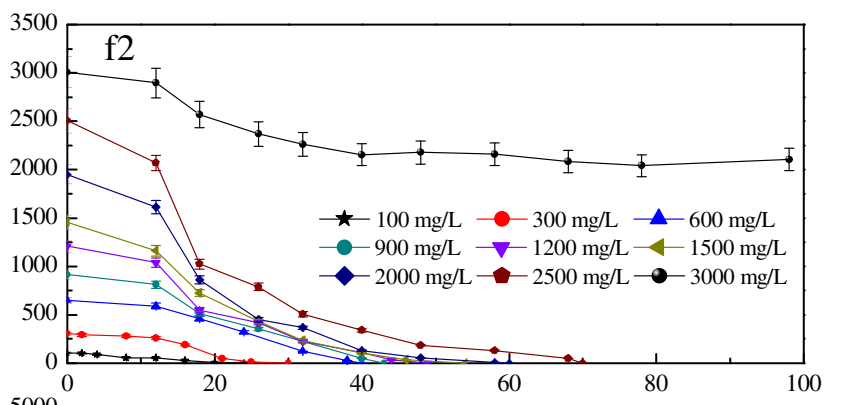

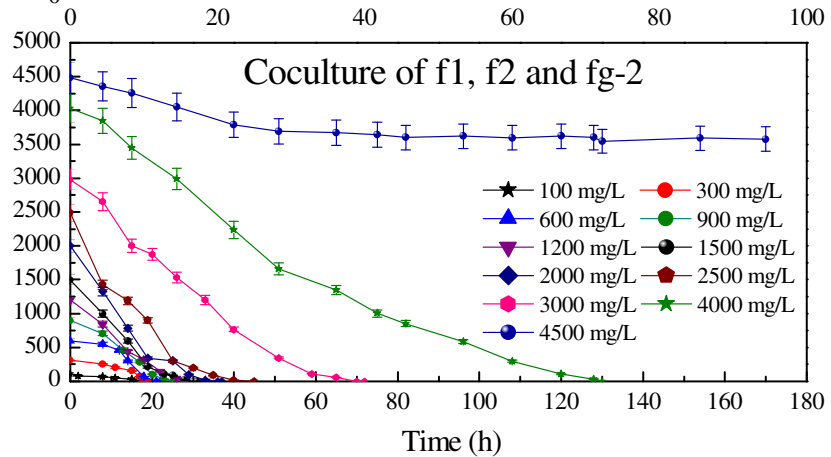

b

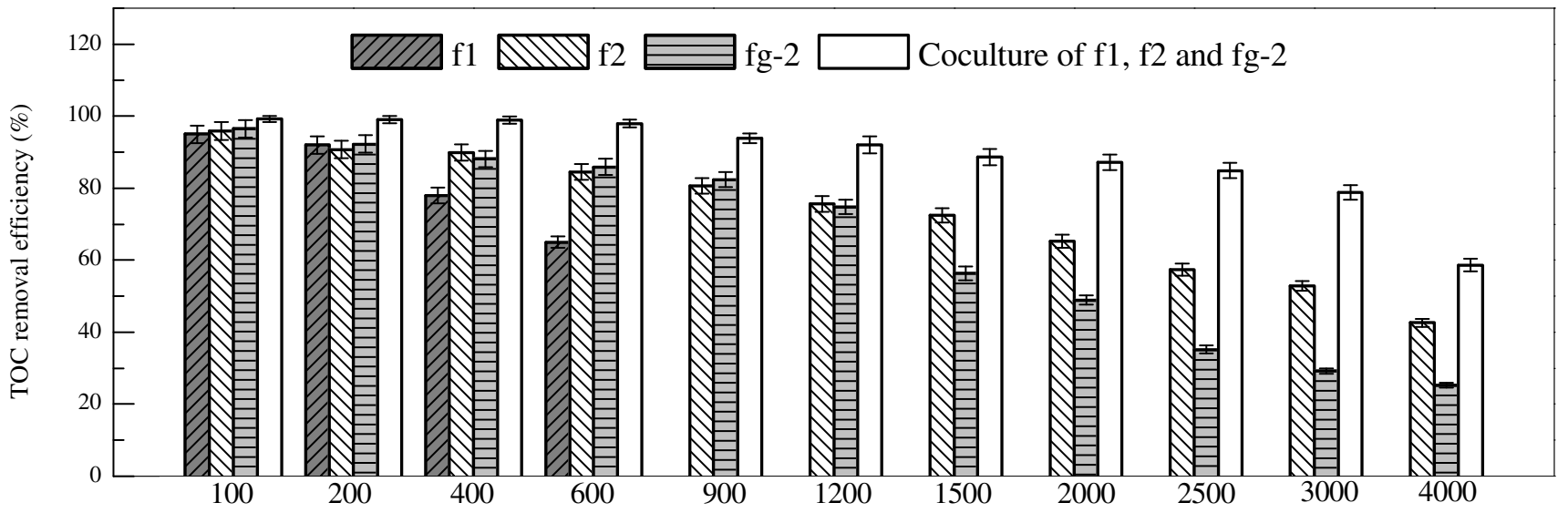

Paracetamol concentration $(\mathrm{mg} / \mathrm{L})$

Fig. 1 Degradation profile and mineralization of paracetamol. a Degradation of paracetamol by strains $\mathrm{fl}, \mathrm{f} 2$, and fg- 2 and the co-culture of $\mathrm{f} 1, \mathrm{f} 2$, and fg-2, indicated by consumption of paracetamol with different initial concentrations. b Mineralization extent of paracetamol

as the effects of substrate inhibition became more pronounced. Comparing the growth kinetics of each isolated strain and their consortium utilizing paracetamol, maximum growth rate $\left(\mu_{\max }\right)$ was in the order of the co-culture $>$ fg- $2>$ $\mathrm{f} 2>\mathrm{f} 1$. The specific growth rate obtained was consistent with the degradation pattern seen in Fig. 1a, where the consortium performed much better than each individual strain on the paracetamol degradation. The good agreement between the experimental data and model predictions indicate that the kinetics of paracetamol utilization by the isolated strains and their consortium are adequately described by the Haldane modification of the Monod equation. biodegradation by strains $\mathrm{f} 1, \mathrm{f} 2$, and fg- 2 and the co-culture of $\mathrm{f} 1, \mathrm{f} 2$, and fg-2, indicated by TOC removal efficiencies with different initial concentrations. Data points are given as means \pm standard deviations $(n=3)$

\section{Oxygen consumption}

Rates of oxygen consumption by washed cell suspensions of strains $\mathrm{fl}, \mathrm{f} 2$, and fg-2 and their consortium grown on paracetamol were recorded at a constant concentration of dissolved oxygen $(7.3 \mathrm{mg} / \mathrm{L}$ final $)$ and initiated by adding various amounts of paracetamol (100 to $4,000 \mathrm{mg} / \mathrm{L}$ final). Compared to each isolated strain, the consortium had the higher oxygen utilization rate (OUR) values at all cases. The specific OURs ranged from 0.304 to $1.78 \mathrm{mg} \mathrm{O}_{2} / \mathrm{mg}$ (biomass) $\mathrm{h}$ that was about $10.2-33.5,2.8-3.4$, and $2.6-2.8$ times that of $\mathrm{fl}, \mathrm{f} 2$, and fg-2, respectively (Fig. 3). For all 

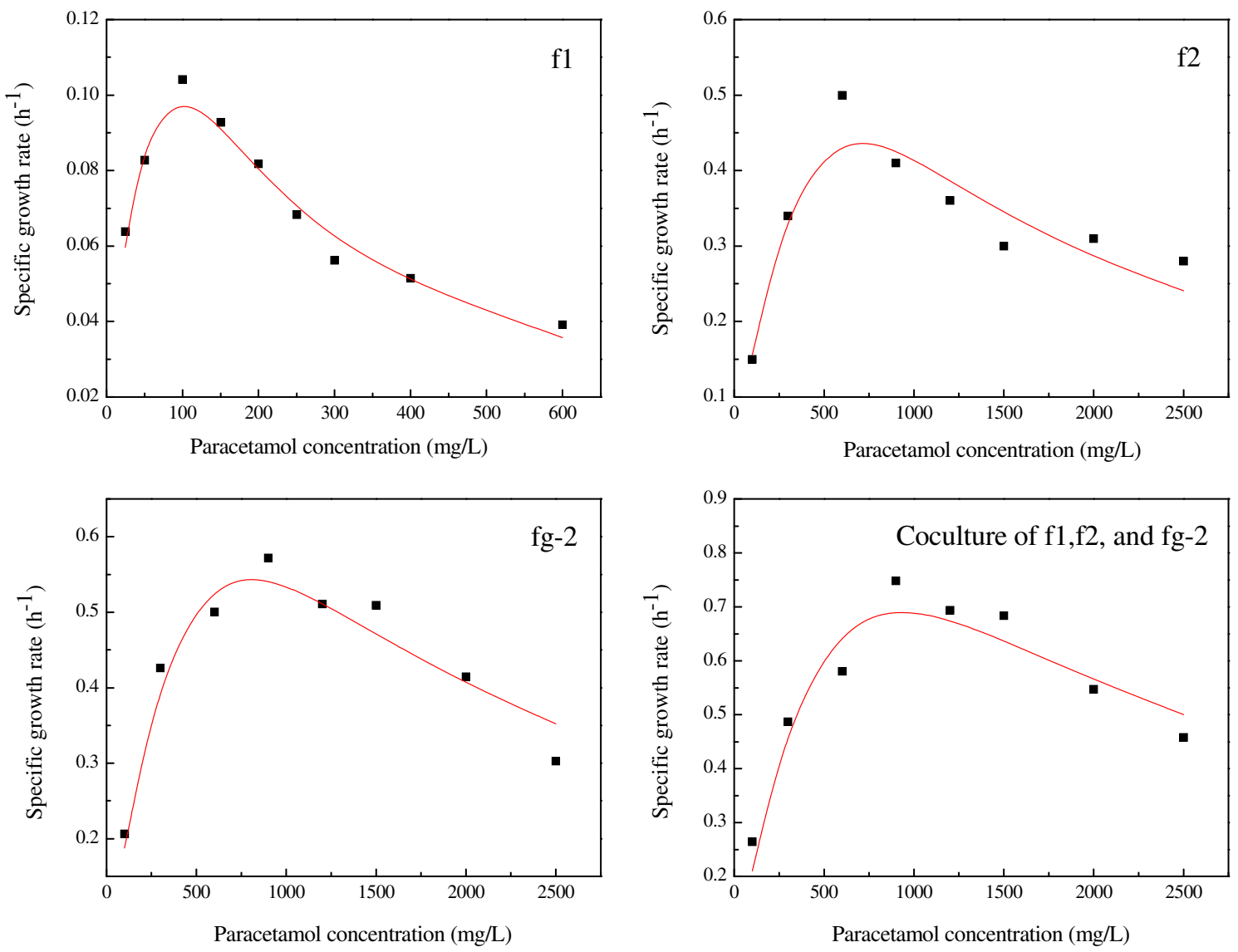

Fig. 2 Growth kinetics of strains $\mathrm{f} 1, \mathrm{f} 2$, and fg- 2 and the co-culture of $\mathrm{fl}, \mathrm{f} 2$, and fg-2. Points represent the experimental results and the lines the fitted kinetics model

three strains and their consortium, the specific OURs at the different initial concentrations of paracetamol correlated reasonably well with the degradation capacity and specific growth rates we reported above.

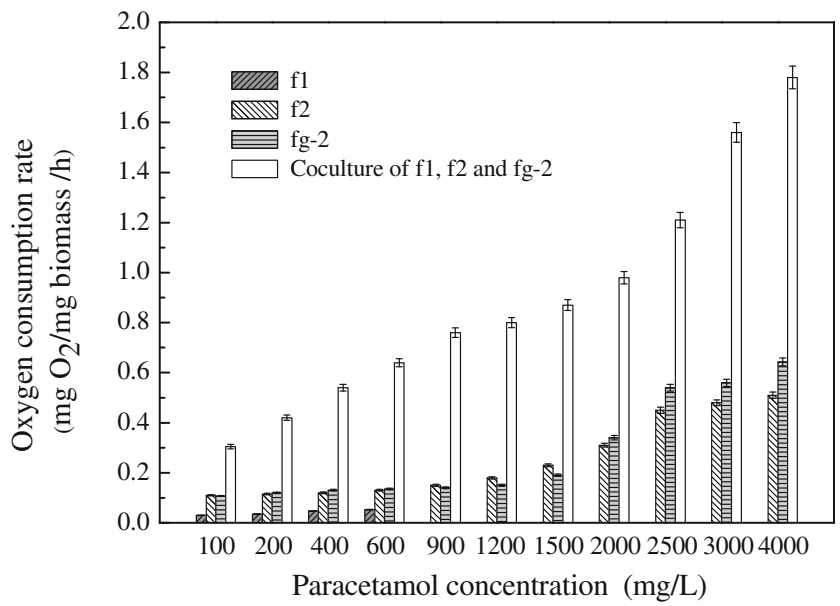

Fig. 3 Rates of oxygen consumption by washed cell suspensions of strains $\mathrm{fl}, \mathrm{f} 2$, and fg- 2 and the co-culture of $\mathrm{f} 1, \mathrm{f} 2$, and fg- 2 grown on paracetamol with different initial concentrations. Data points are given as means \pm standard deviations $(n=3)$
Possible intermediate

The identities of metabolic intermediates were confirmed by GC-MS analysis of TMS derivatives by comparison with commercially available authentic standards and by matching against a mass spectra library. The three isolated strains, f1, $\mathrm{f} 2$, and fg-2, generated almost the same metabolic products in the aerobic process. Analysis of GC-MS chromatograms of derivatized samples revealed two major peaks for paracetamol metabolite compounds at $16.470 \mathrm{~min}$ (A) and $18.211 \mathrm{~min}(\mathrm{~B})$ on the total ion chromatogram (See Fig. S2, ESM). Their mass spectra and the possible fragments during GC-MS analysis for these products are shown in Fig. 4. The metabolite A had a molecular ion at $\mathrm{m} / \mathrm{z} 254$ and prominent ions due to fissions of $\cdot \mathrm{CH}_{3}, \cdot \mathrm{OS}_{\mathrm{i}}\left(\mathrm{CH}_{3}\right)_{3}$ and $\cdot \mathrm{S}_{\mathrm{i}}\left(\mathrm{CH}_{3}\right)_{3}$ at $m / z 239,166$, and 181 , respectively, suggesting that the metabolite is derivatized hydroquinone, as expected (Fig. 4a). Further confirmation of this compound as hydroquinone was obtained by comparison of its mass spectrum with that of authentic derivatized hydroquinone. The metabolite $\mathrm{B}$ had a molecular ion at $\mathrm{m} / \mathrm{z} 181$ and significant fragment ions at $m / z 166,108,150,138$, and 73 , which suggests that this metabolite is derivatized 4-aminophenol. Peak B had the same 

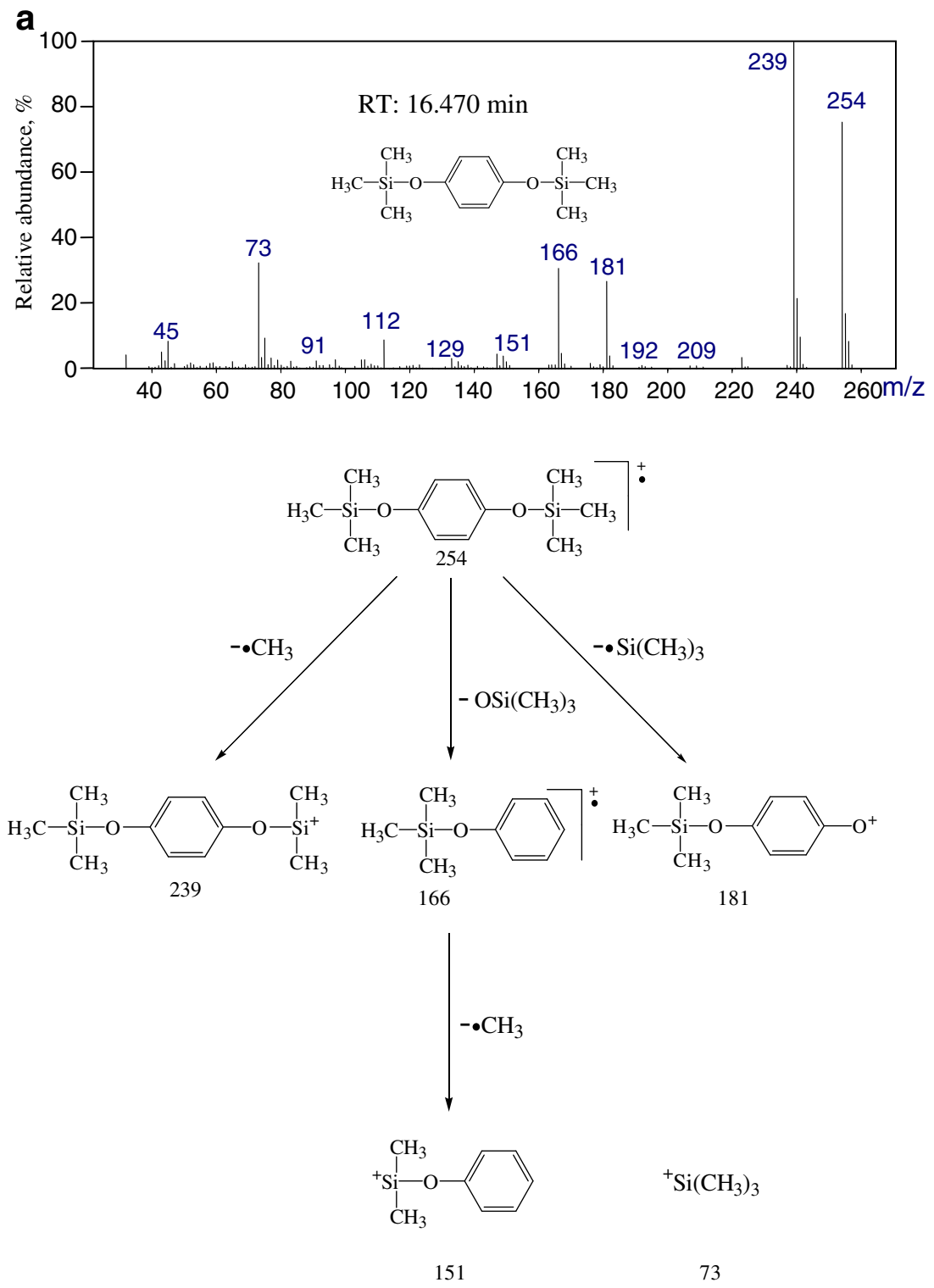

Fig. 4 Concentration profiles of paracetamol and identified main intermediates during the biodegradation of paracetamol with an initial concentration of $2,000 \mathrm{mg} / \mathrm{L}$. a f1, $\mathbf{b} \mathrm{f} 2, \mathbf{c}$ fg- 2 , $\mathbf{d}$ the co-culture of $\mathrm{fl}, \mathrm{f} 2$, and fg-2. Data points are given as means \pm standard deviations $(n=3$ )

retention time and mass spectrum as an authentic sample of derivatized 4-aminophenol. 4-Aminophenol was also indentified as a main intermediate during photocatalytic degradation of paracetamol (Yang et al. 2009).

The main metabolic intermediates were also determined by HPLC-MS (see Fig. S-3, ESM). HPLC chromatograms reveal the formation of two metabolites. Compounds 1 and 2 presented mass spectra with two major ions at $\mathrm{m} / \mathrm{z} 108$ and 109 , which corresponded to a molecular mass $[\mathrm{M}-\mathrm{H}]^{-}$of 4aminophenol and hydroquinone, respectively.

To provide additional evidence for 4-aminophenol and hydroquinone as main intermediates of paracetamol biodegradation, biodegradation of these compounds as the sole substrate for growth of strains $\mathrm{f} 1, \mathrm{f} 2$, and fg- 2 was tested as well (see Table 1-S, ESM). It is clear that these paracetamolgrown cells of $\mathrm{f1}, \mathrm{f} 2$, and fg-2 were able to degrade 4aminophenol and hydroquinone as the sole growth substrate with a different exponential growth rate. These data make paracetamol metabolism mainly through 4-aminophenol and hydroquinone more likely.

Some carboxylic acids and inorganics were determined by GC-MS and IC analysis, as listed in Table 1. Following the two aromatic intermediate compounds, five carboxylic acids were successfully isolated and identified, suggesting that the two aromatic compounds could be precursors of these carboxylic acids. Nitrite and nitrate could be detected, indicating $-\mathrm{CO}-\mathrm{NH}_{2}$ group of paracetamol could be easily mineralized to nitrite and nitrate by the isolated strains. The 
Fig. 4 (continued)
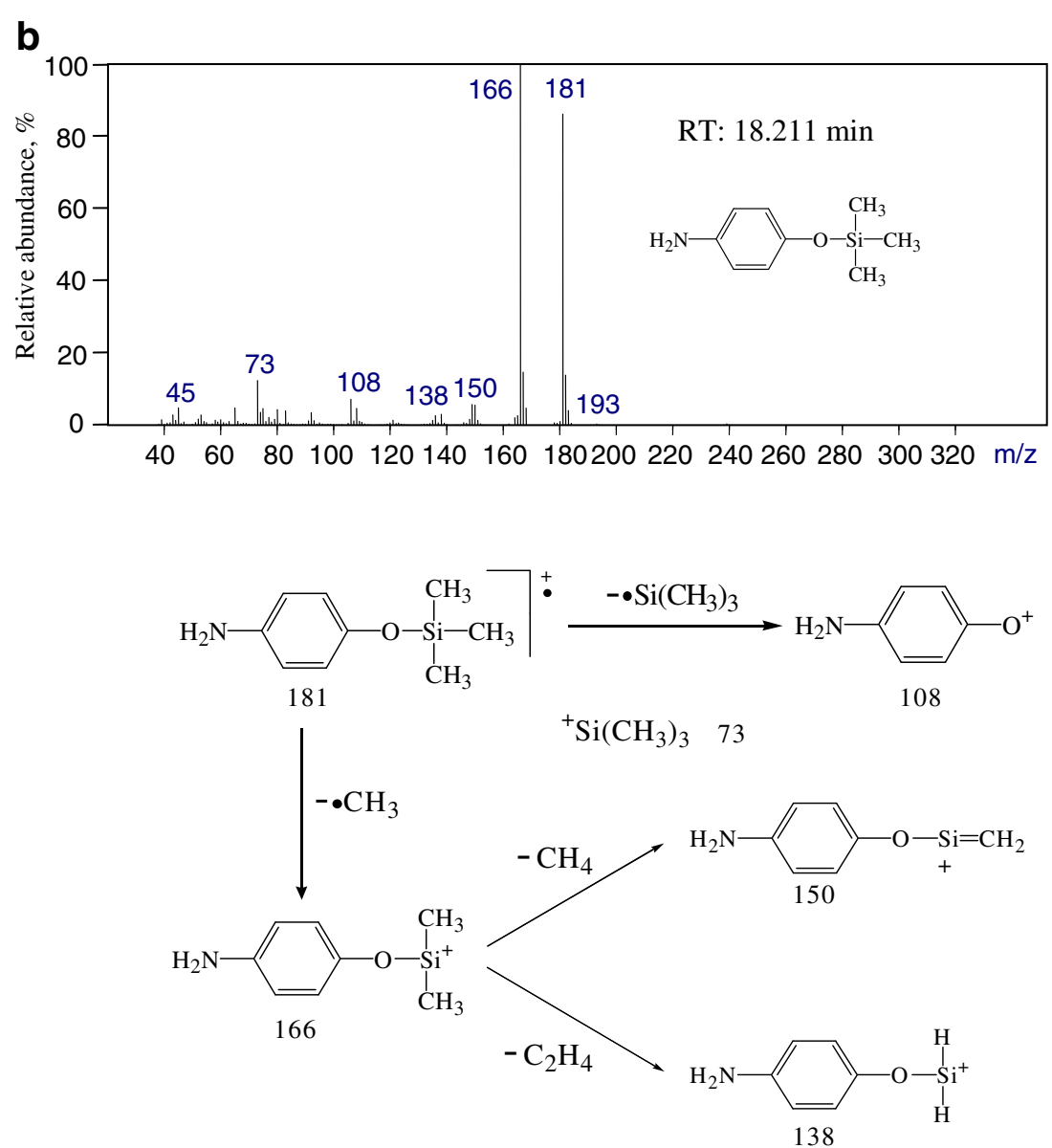

observed nitrite and nitrate could also be taken as an indicator for the biodegradation and mineralization of paracetamol.

The concentration profiles of the main metabolic intermediates during the biodegradation of paracetamol by the isolated strains and their consortium are shown in Fig. 5. Figure $5 \mathrm{a}$ indicates only $16.3 \%$ of paracetamol with an initial concentration of $2,000 \mathrm{mg} / \mathrm{L}$ was degraded by strain f1. The metabolic intermediate products, 4-aminophenol and hydroquinone, increased rapidly and reached a maximum accumulation of 65.2 and $48.7 \mathrm{mg} / \mathrm{L}$ at 60 and $120 \mathrm{~h}$, respectively. However, the two metabolic intermediates were not further degraded by fl. These results suggested the growth of f1 was inhibited by paracetamol with an initial concentration of $2,000 \mathrm{mg} / \mathrm{L}$ strongly. For f2, the first appeared intermediates were also 4aminophenol and hydroquinone which reached their maximum of concentration (264.0 and $246.8 \mathrm{mg} / \mathrm{L}$ ) around 26 and $32 \mathrm{~h}$ then decreased progressively to disappear from solution after 60 and $64 \mathrm{~h}$, respectively (Fig. 5b). After $45 \mathrm{~h}$ of incubation, no paracetamol was detected in the culture medium of fg-2. Consequently, 4-aminophenol and hydroquinone gradually increased from the beginning and reached a maximum at 25 and $29 \mathrm{~h}$, respectively. After that, the concentration of the two intermediate products decreased gradually to zero at $45 \mathrm{~h}$ (Fig. 5c). For the coculture of f1, f2, and fg-2, 4-aminophenol and hydroquinone were also detected in the culture medium. Relative to the above pure strains, 4-aminophenol and hydroquinone showed lower maximum accumulation concentrations (31.6 and $22.2 \mathrm{mg} / \mathrm{L})$, which occurred around 14 and $19 \mathrm{~h}$, respectively.

\section{Discussion}

Biodegradation of recalcitrant compounds requires the enrichment of suitable microbial culture followed by the determination of its metabolic and kinetic parameters. In the present study, three bacterial organisms, Stenotrophomonas sp. strain $\mathrm{fl}$ and Pseudomonas sp. strains $\mathrm{f} 2$ and fg-2, that can utilize paracetamol for growth are obtained. The three pure cultures were free of requirements for yeast extract or other growth factors. To date, only $D$. tsuruhatensis and $P$. aeruginosa isolated from a membrane bioreactor were discovered able to degrade paracetamol very recently (Gusseme et al. 2011). We, for the first time, found that Stenotrophomonas species could degrade paracetamol as the sole source of 
Table 1 Identified intermediates during degradation of paracetamol using GC-MS and IC

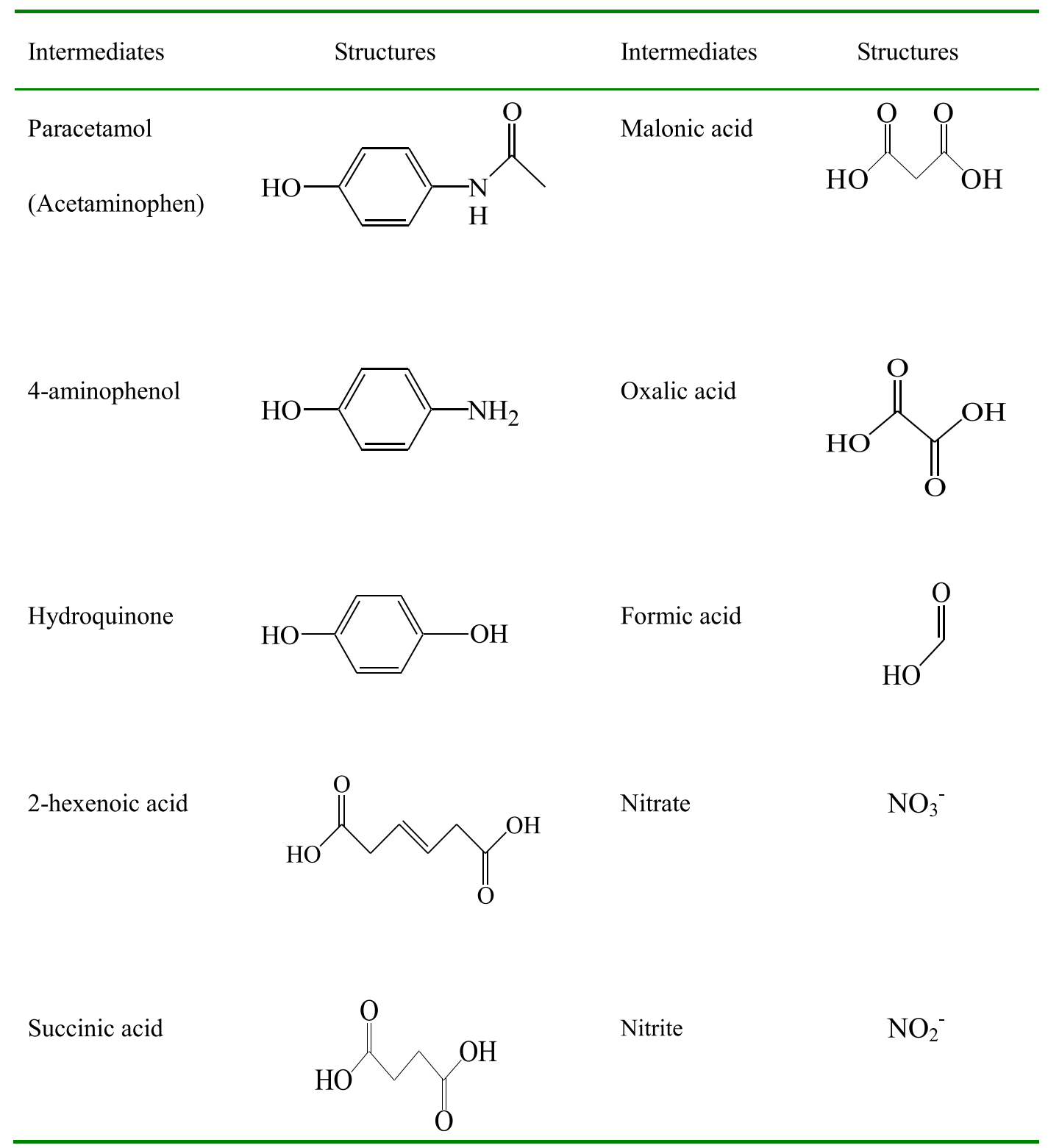

carbon, nitrogen, and energy. Members of the Pseudomonas are environmental organisms known for their ability to degrade aromatic compounds of environmental concern (Cámara et al. 2009; Neumann et al. 2004). However, except for P. aeruginosa (Gusseme et al. 2011), the genus has not included any known paracetamol degraders until now.

In our work, the three organisms were grown as monospecies and as a mixed-species culture with paracetamol as the sole carbon, nitrogen, and energy source. Our data show that $\mathrm{fl}, \mathrm{f} 2$, and $\mathrm{fg}-2$ could perform complete degradation of paracetamol at concentrations of 400, 2,500, and 2,000 mg/ L or below, respectively. As described in Fig. 1, f1, f2, and fg-2 differed significantly in their capacity of paracetamol degradation. The fg-2 degraded paracetamol with a much higher rate than the other two organisms, but showed the longest lag phase before degradation. The $\mathrm{f} 2$ could tolerate a higher paracetamol concentration of up to $2,500 \mathrm{mg} / \mathrm{L}$, implying its best resistance to paracetamol among these strains. Although compared to the other two isolates f1 showed a much lower degradation rate and poor resistance to paracetamol, it reduced the lag time before degradation greatly. The results obtained from the degradation assays allowed us to show that the three isolated bacteria possibly played a major role in the paracetamol removal.

It is possible to compare degradation capacity between the isolated pure strains and their consortium here because the same conditions were controlled. When these three strains were incubated together, as shown in Figs. 1, 2, and 3 , a co-culture was observed to have better degradation characteristics as compared to the use of individual strains. 
Fig. 5 Mass spectra of the major TMS-derivatized metabolites isolated from culture fluids during growth of strains grown on paracetamol a

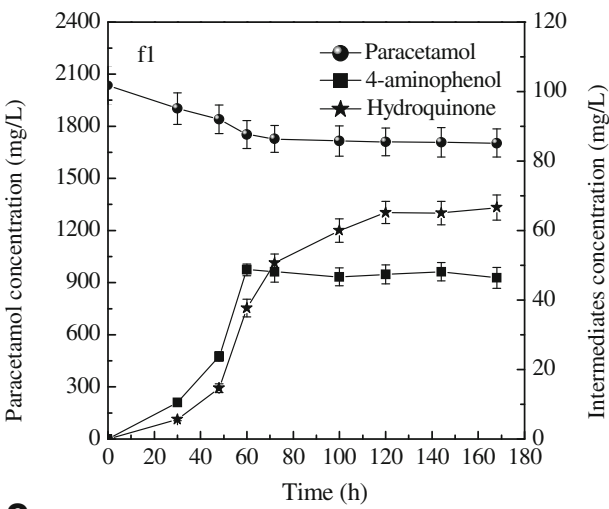

C

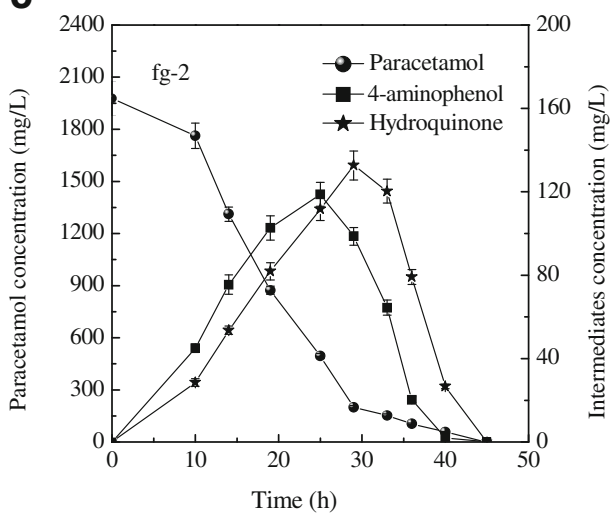

b

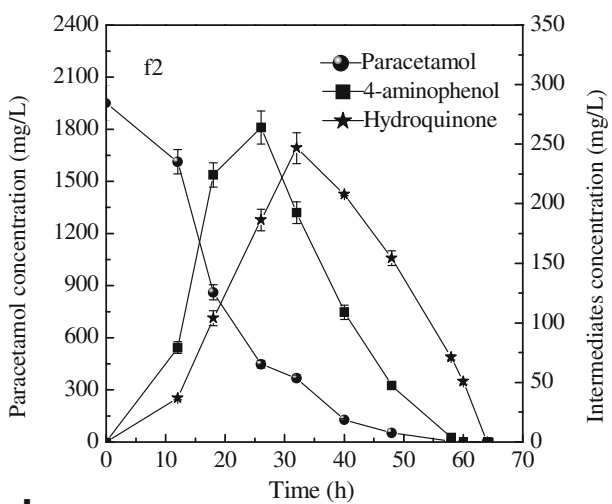

d

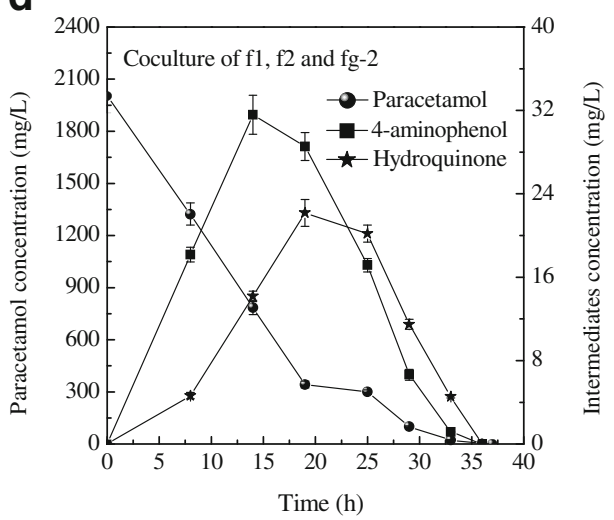

The consortium achieved substantially higher degradation rates and significantly better tolerance to paracetamol with a shorter lag time. Furthermore, the co-culture mineralized significantly more paracetamol than did pure strains in all cases and to various extents. A combination of three microbial strains recovered from the enriched culture was shown to be necessary for complete paracetamol mineralization. The data suggested a possible complementary interaction among the various isolates of the consortium. The oxygen consumption rates of the consortium were also consistently greater than that of the pure cultures at the all measured concentrations of paracetamol (Fig. 3), further suggesting the cooperative degradation and mineralization capacities of these three isolates. Thus, the degradation of paracetamol could be performed by the single isolates, but is greatly stimulated by a synergistic interaction of the consortium obtained from the same paracetamol-degrading culture. In fact, microbial mixed cultures are generally considered to be potentially more efficient in the biodegradation of recalcitrant compounds (Kim et al. 2009). A co-culture of three different bacterial species was shown to be necessary for complete fluorobenzene mineralization (Carvalho et al. 2002). Joshi et al. (2008) reported acid orange decolorization was significantly higher with the use of consortium as compared to the use of individual strains. Bacterial cocultures have been used to increase the rate of degradation of phenol (Jiang et al. 2006) and diuron (Sørensen et al. 2008). In the latter case, the combination of Arthrobacter
Fig. 6 Proposed pathway for biodegradation of paracetamol

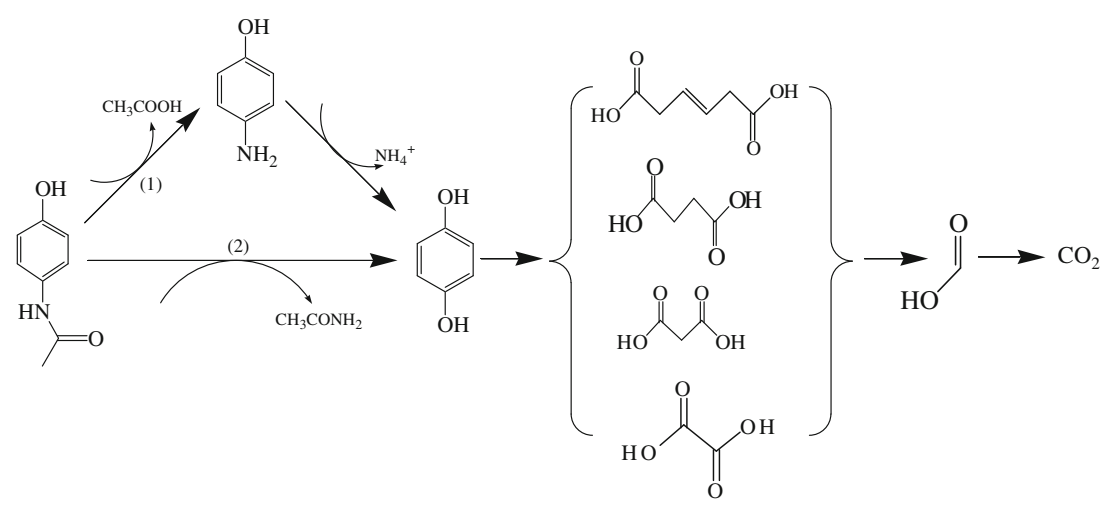


globiformis strain D47 and Variovorax sp. strain SRS16 resulted in mineralization of diuron to a greater extent.

In order to allow rational design of biodegradation systems for paracetamol-contaminated wastewater, it is necessary to investigate the metabolic pathway to allow evaluation of whether toxic intermediates can be expected to accumulate. The metabolites of the initial steps in the paracetamol catabolic pathway were conclusively identified as 4-aminophenol and hydroquinone by GC-MS and HPLC-MS. Based on the above results, we propose the following pathway for paracetamol degradation (Fig. 6): (1) paracetamol is initially catalyzed by an amidohydrolase with the release of acetate to yield 4-aminophenol, in which the amino group is then replaced by a hydroxyl group to form hydroquinone, subsequent to ring fission. (2) In the second instance, the initial hydroxylation of paracetamol could potentially be catalyzed by a hydrolytic enzyme to produce hydroquinone with a release of acetamide, which could be further converted into oxamic acid. However, attempts to isolate and identify acetamide and oxamic acid failed. Strains that converted aminophenol to the corresponding 1,4-benzenediol or hydroquinone compounds have been described. Burkholderia sp. strain AK-5 could possibly transform 4-aminophenol to hydroquinone (Murakami et al. 2003). Pseudomonas putida strain TW3 utilized 4-hydroxylaminobenzoate lyase oxidized 4hydroxylaminobenzoate to protocatechuate, replacing the amino group by a hydroxyl group (Hughes et al. 2002). Following the aromatic compounds, some carboxylic acids were detected by GC-MS and IC analysis, suggesting the aromatic compounds can be the precursors of the carboxylic acids. To our knowledge, this is the first description of the biodegradation pathway for paracetamol. The initial steps are partially different from the reactions previously described for photocatalytic and electro-Fenton degradation of paracetamol (Brillas et al. 2009; Yang et al. 2009), in which hydroquinone has been identified as the main transformation products. Gusseme et al. (2011) also reported that hydroquinone was a temporary transformation product of paracetamol degradation by two bacterial strains, $D$. tsuruhatensis and P. aeruginosa.

Our results demonstrate that the three isolated strains can grow on paracetamol as sole carbon, nitrogen, and energy source. Paracetamol conversion was proposed to proceed via 4-aminophenol to hydroquinone, which is the major route in the isolated strains. A combination of the three microbial strains recovered from the same culture was shown to be necessary for complete paracetamol degradation and mineralization, suggesting a possible complementary interaction among the various isolates. The exact roles of each of the strains in the consortium and the cooperative mechanism involved in the degradation of paracetamol are still unknown and are being further investigated.
Acknowledgments This work was supported by the National Natural Science Foundation of China (no. 50908210 and 21177116) and the Important Provincial Science \& Technology Specific Project (no. 2011C13023) from Zhejiang Province, China.

\section{References}

Andreozzi R, Caprio V, Marotta R, Vogna D (2003) Paracetamol oxidation from aqueous solutions by means of ozonation and $\mathrm{H}_{2} \mathrm{O}_{2} / \mathrm{UV}$ system. Water Res 37(5):993-1004

Benotti MJ, Trenholm RA, Vanderford BJ, Holady JC, Stanford BD, Snyder SA (2009) Pharmaceuticals and endocrine disrupting compounds in U.S. drinking water. Environ Sci Technol 43:597603

Brillas E, Sirés I, Arias C, Cabot PL, Centellas F, Rodríguez RM, Garrido JA (2005) Mineralization of paracetamol in aqueous medium by anodic oxidation with a boron-doped diamond electrode. Chemosphere 58(4):399-406

Brillas E, Sirés I, Oturan MA (2009) Electro-Fenton process and related electrochemical technologies based on Fenton's reaction chemistry. Chem Rev 109:6570-6631

Cámara B, Nikodem P, Bielecki P, Bobadilla R, Junca H, Pieper DH (2009) Characterization of a gene cluster involved in 4chlorocatechol degradation by Pseudomonas reinekei MT1. J Bacteriol 191(15):4905-4915

Carvalho MF, Alves CCT, Ferreira MIM, De Marco P, Castro PML (2002) Isolation and initial characterization of a bacterial consortium able to mineralize fluorobenzene. Appl Environ Microbiol 68(1):102-105

Chen CY, Chen SC, Fingas M, Kao CM (2010) Biodegradation of propionitrile by Klebsiella oxytoca immobilized in alginate and cellulose triacetate gel. J Hazard Mat 177:856-863

Escher BI, Baumgartner R, Koller M, Treyer K, Lienert J, McArdell CS (2011) Environmental toxicology and risk assessment of pharmaceuticals from hospital wastewater. Water Res 45:75-92

Gusseme BD, Vanhaecke L, Verstraete W, Boon N (2011) Degradation of acetaminophen by Deftia tsuruhatensis and Pseudomonas aeruginosa in a membrane bioreactor. Water Res 45:18291837

Hasan SA, Ferreira MIM, Koetsier MJ, Arif MI, Janssen DB (2011) Complete biodegradation of 4-fluorocinnamic acid by a consortium comprising Arithrobacter sp. strain G1 and Ralstonia sp. strain H1. Appl Environ Microbiol 77(2):572-579

Hu J, Zhou L, Zhou QW, Wei F, Zhang LL, Chen JM (2012) Biodegradation of paracetamol by aerobic granules in a sequencing batch reactor (SBR). Adv Mater Res 441:531-535

Huber MM, Göbel A, Joss A, Hermann N, Löffler D, Mcardell CS, Ried A, Siegrist H, Ternes TA, Gunten UV (2005) Oxidation of pharmaceuticals during ozonation of municipal wastewater effluents: a pilot study. Environ Sci Technol 39:4290-4299

Hughes MA, Baggs MJ, Al-Dulayymi J, Baird MS, Williams PA (2002) Accumulation of 2-aminophenoxazin-3-one-7-carboxylate during growth of Pseudomonas putida TW3 on 4-nitro-substituted substrates requires 4-hydroxylaminobenzoate lyase (PnbB). Appl Environ Microbiol 68:4965-4970

Jiang HL, Tay JH, Maszena AM, Tay STL (2006) Enhanced phenol biodegradation and aerobic granulation by two coaggregating bacterial strains. Environ Sci Technol 40:6137-6142

Joshi T, Iyenga L, Singh K, Garg S (2008) Isolation, identification and application of novel bacterial consortium TJ-1 for the decolourization of structurally different azo dyes. Bioresour Technol 99:7115-7121 
Kim YM, Ahn CK, Woo SH, Jung GY, Park JM (2009) Synergic degradation of phenanthrene by consortia of newly isolated bacterial strains. J Biotechnol 144:293-298

Kolpin DW, Furlong ET, Meyer MT, Thurman EM, Zaugg SD, Barben LB, Buxton HT (2002) Pharmaceuticals, hormones, and other organic wastewater contaminants in U.S. streams, 1999-2000: a national reconnaissance. Environ Sci Technol 36(6):1202-1211

Langford KH, Thomas KV (2009) Determination of pharmaceutical compounds in hospital effluents and their contribution to wastewater treatment works. Environ Int 25:766-770

Li TG, Liu JX, Bai RB, Ohandja DG, Wong F-S (2007) Biodegradation of organonitriles by adapted activated sludge consortium with acetonitrile-degrading microorganisms. Water Res 41:3465-3473

Murakami S, Aoki K, Takenake S, Okugawa S, Kadowaki M (2003) The metabolic pathway of 4-aminophenol in Burkholderia sp. strain AK-5 differs from that of aniline and aniline with C-4 substituents. Appl Environ Microbiol 69(9):5410-5412

Neumann G, Teras R, Monson L, Kivisaar M, Schauer F, Heipieper HJ (2004) Simultaneous degradation of atrazine and phenol by Pseudomonas sp. strain ADP: effect of toxicity and adaptation. Appl Environ Microbiol 70(4):1907-1912

Philips PJ, Smith SG, Koplin DW, Zaugg SD, Buxton HT, Furlong ET, Esposito K, Stinson B (2010) Pharmaceutical formulation facilities as sources of opioids and other pharmaceuticals to wastewater treatment plant effluents. Environ Sci Technol 44:4910 4916
Robert PH, Thomas KV (2006) The occurrence of selected pharmaceuticals in wastewater effluent and surface waters of the lower Tyne catchment. Sci Total Environ 356(1-3):143-153

Rosal R, Rodríguez Perdigón-Melón JA, Mezcua M, Hernando MD, Letón P, García-Calvo E, Agüera A, Fernández-Alba AR (2008) Removal of pharmaceuticals and kinetics of mineralization by $\mathrm{O}_{3} /$ $\mathrm{H}_{2} \mathrm{O}_{2}$ in a biotreated municipal wastewater. Water Res 42:3719-3728

Shariati FP, Mehrnia MR, Salmasi BM, Heran M, Wisniewski C, Sarrafzadeh MH (2010) Membrane bioreactor for treatment of pharmaceutical wastewater containing acetaminophen. Desalination 250:798-800

Skoumal M, Cabot P-L, Centellas F, Arias C, Rodríguez RM, Garrido JA, Brillas E (2006) Mineralization of paracetamol by ozonation catalyzed with $\mathrm{Fe}^{2+}, \mathrm{C}_{\mathrm{U}}^{2+}$ and UVA light. Appl Catal B: Environ 66:228-240

Sørensen SR, Albers CN, Aamand J (2008) Rapid mineralization of the phenylurea herbicide diuron by Variovorax sp. strain SRS 16 in pure culture and within a two-member consortium. Appl Environ Microbiol 74(8):2332-2340

Ternes TA (1998) Occurrence of drugs in German sewage treatment plants and rivers. Water Res 32(11):3245-3260

Yang LM, Yu LE, Ray MB (2008) Degradation of paracetamol in aqueous solutions by $\mathrm{TiO}_{2}$ photocatalysis. Water Res 42:2480-2488

Yang LM, Yu LE, Ray MB (2009) Photocatalytic oxidation of paracetamol: dominant reactants, intermediates, and reaction mechanisms. Environ Sci Technol 43:460-465 